Methodology article

Open Access

\title{
Optimization of candidate-gene SNP-genotyping by flexible oligonucleotide microarrays; analyzing variations in immune regulator genes of hay-fever samples Janne Pullat ${ }^{1,3,5}$, Robert Fleischer ${ }^{1}$, Nikolaus Becker ${ }^{2}$, Markus Beier ${ }^{4}$, Andres Metspalu $3,5,6,7$ and Jörg D Hoheisel*1
}

Address: ${ }^{1}$ Division of Functional Genome Analysis, Deutsches Krebsforschungszentrum, Im Neuenheimer Feld 580, 69120 Heidelberg, Germany, ${ }^{2}$ Division of Clinical Epidemiology, Deutsches Krebsforschungszentrum, Im Neuenheimer Feld 580, 69120 Heidelberg, Germany, ${ }^{3}$ Institute of Molecular and Cell Biology, 23 Riia St., 51010 Tartu, Estonia, ${ }^{4}$ Febit biotech, Im Neuenheimer Feld 517, 69120 Heidelberg, Germany, ${ }^{5}$ The Estonian Biocentre, 23b Riia St., 51010 Tartu, Estonia, ${ }^{6}$ Molecular Diagnostics Centre, United Laboratories of the Tartu University Hospital, $1 \mathrm{a}$ Puusepa Str., 50406 Tartu, Estonia and 7Estonian Genome Project of University of Tartu, 61b Tiigi Str., 50410 Tartu, Estonia

Email: Janne Pullat - janne@ut.ee; Robert Fleischer - Robert.Fleisher@gmx.de; Nikolaus Becker - n.becker@dkfz-heidelberg.de; Markus Beier - markus.beier@febit.de; Andres Metspalu - Andres.Metspalu@ebc.ee; Jörg D Hoheisel* - j.hoheisel@dkfz-heidelberg.de

* Corresponding author

Published: 17 August 2007

BMC Genomics 2007, 8:282 doi:10.1/86/147|-2164-8-282
Received: 20 November 2006

Accepted: 17 August 2007

This article is available from: http://www.biomedcentral.com/I47I-2/64/8/282

(c) 2007 Pullat et al; licensee BioMed Central Ltd.

This is an Open Access article distributed under the terms of the Creative Commons Attribution License (http://creativecommons.org/licenses/by/2.0), which permits unrestricted use, distribution, and reproduction in any medium, provided the original work is properly cited.

\begin{abstract}
Background: Genetic variants in immune regulator genes have been associated with numerous diseases, including allergies and cancer. Increasing evidence suggests a substantially elevated disease risk in individuals who carry a combination of disease-relevant single nucleotide polymorphisms (SNPs). For the genotyping of immune regulator genes, such as cytokines, chemokines and transcription factors, an oligonucleotide microarray for the analysis of 99 relevant SNPs was established. Since the microarray design was based on a platform that permits flexible in situ oligonucleotide synthesis, a set of optimally performing probes could be defined by a selection approach that combined computational and experimental aspects.

Results: While the in silico process eliminated $9 \%$ of the initial probe set, which had been picked purely on the basis of potential association with disease, the subsequent experimental validation excluded more than twice as many. The performance of the optimized microarray was demonstrated in a pilot study. The genotypes of 19 hay-fever patients (aged 40-44) with high IgE levels against inhalant antigens were compared to the results obtained with 19 age- and sexmatched controls. For several variants, allele-frequency differences of more than $10 \%$ were identified.
\end{abstract}

Conclusion: Based on the ability to improve empirically a chip design, the application of candidateSNP typing represents a viable approach in the context of molecular epidemiological studies.

\section{Background}

Array-based technologies are revolutionizing genomics, especially the analysis of DNA variation. Array technolo- gies are not without limitations, however, and one major drawback is the poor flexibility of typical array formats. It is cumbersome to create one's own tailored arrays by spot- 
ting DNA. Commercially available microarrays, on the other hand, either contain a fixed and usually broadly applicable content or are expensive to purchase with customized features. The fixed-content arrays are useful for taking advantage of the high resolution genetic map of the human genome that is based on single nucleotide polymorphisms $[1,1]$, which define DNA blocks (haplotypes) [1]. Since SNPs are the most common type of genetic variation between individuals, it makes sense to utilize them for the localization of disease genes by identifying haplotypes that are associated with phenotypic traits, especially in the case of multifactorial diseases [1-5]. As a consequence of such a study, however, further analysis is required for improving the resolution of the mapping process or trying to identify the polymorphisms that are actually responsible for the phenotypic variation. Alternatively to the process described above, one can immediately focus on the analysis of particular polymorphisms in candidate genes, if circumstantial evidence indicates their possible relevance to the occurrence of a disease. In either approach the production of a customized microarray is required. Also, experience has demonstrated the need for a careful design of the experimental set-up in order to avoid unacceptable error [6].

Irrespective of the algorithm used for the sequence selection of the probe set, the final functional test of the suitability of an oligonucleotide array for genotyping results from an empirical analysis of the hybridization performance of the oligonucleotide probes. In consequence, it is likely that the initial chip design will be changed by replacing ill-performing oligonucleotides with alternative sequences. For this process, the ability to easily change the chip layout is essential. Light-induced in situ synthesis controlled by a micro-mirror device $[7,8]$ combines high synthesis yields of more than $99.5 \%$ per condensation [9] - and therefore good oligonucleotide quality - with the power of producing oligomer arrays of high density, reproducible characteristics and flexible layout.

In this study, we present the process of establishing an oligonucleotide microarray based on an on-site in situ synthesis technology for typing DNA samples in immune regulator genes including cytokines, chemokines and transcription factors. Genetic variants in immune regulator genes have been associated with numerous diseases, including allergies and cancer, with apparently an elevated disease risks in individuals that carry a combination of disease-relevant SNPs. For the array design, we exploited the flexibility of the GeniomOne device [8]. It employs a digital projector to synthesize oligonucleotide array features within channels of a three-dimensional micro-fluidic reaction carrier. The system allows the synthesis of a probe set of up to 64,000 oligonucleotides on a single chip, which subsequently can be hybridized with up to eight samples. For this analysis a microarray that assays 99 relevant SNPs was established by an iterative cycle of probe design and experimental evaluation. Subsequently, the performance of this microarray was investigated in a pilot study. Hay-fever patients aged 40-44 that exhibited high IgE levels against inhalant antigens and an age and sex-matched control group were analyzed.

\section{Results}

From a case-control study on hay-fever [10], 19 cases with the most extreme plasma IgE levels against inhalant antigens and 19 age- and sex-matched non-atopic controls were selected for the project. Originally, 141 SNPs in cytokine genes and other immune regulatory factors were selected from published studies and SNP-databases [1113]. If possible, SNPs with known or potential functional relevance and allele frequency information were selected. Also, sequence complexity between the probes was meant to be similar, since it is well established that the rate of reassociation depends on sequence complexity [14]. In addition, the initial compilation was based on theoretical calculations of interactions between all oligonucleotide probes and PCR fragments. The program "SNP CrossChecker" by Febit GmbH was used to check the cross reactivity between oligoprobes and template sequences reducing the number of PCR-products by 13 to 128 . The threshold of maximally possible homology between 23 mere oligoprobe and template sequences was set to $85 \%$. It takes into account that if within the 23 nucleotides of a probe, 20 nucleotides will basepairing with a template, this will produce sufficiently stable complex to produce false positive signals in the genotyping analysis.

Theoretically the probe properties could be assessed basis their sequence similarity and hybridization properties. Experimentally "bad" probe has low specificity, sensitivity and uniformity under given reaction conditions (temperature, base composition, salt concentration, hybridization time). Specificity and stability of DNA duplex formation strongly depend on sequence and base composition $[15,16]$. Also, the target sequence on either side of the SNP position plays an important role since secondary structures may strongly affect the hybridization behavior of a sample [17]. Therefore, it is frequently insufficient to predict hybridization performance merely on the basis of theoretical calculations. Consequently, we analyzed and optimized the experimental parameters of SNP position in the oligonucleotide and the overall length of the probes as well as hybridization temperature and duration. For each SNP, all four possible sequence variations were applied to the chip. One of the probes is designed to be perfectly complementary to a short stretch of the reference sequence (perfect match - PM) and the other three are identical to the first, except at the interrogation position, where one of the other three bases is substituted (mis- 


\begin{abstract}
tct tgc aaact aagaaagcacac aca accttgacetgtggct tctgctgttcc ccagcactgtcgce atgtgctgtcagcagcagcactctccccgactggcacat ccc agt gga agttgtgceggacagcaccagtgatctgt aca acttccaggtgt cacccat
\end{abstract}

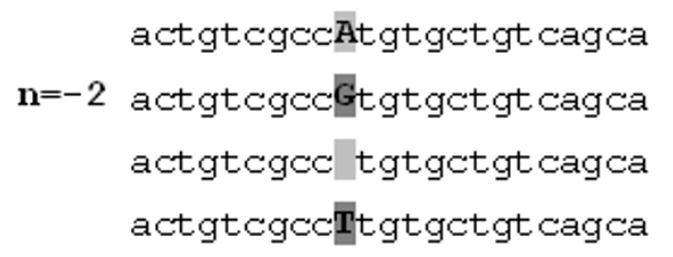
gcactgtcgccAtgtgctgtcag $\mathbf{n}=\mathbf{0}$ gcactgtcgccGtgtgctgtcag gcactgtcgccCtgtgctgtcag gcactgtcgcdTtgt get gtcag cagcactgt cgccAtgtgctgtc
$\mathbf{n =}+2$ cagcactgt cgccGtgtgctgtc
cagcactgt cgccCtgtgctgtc
cagcactgt cgccitgtgctgtc SENSE

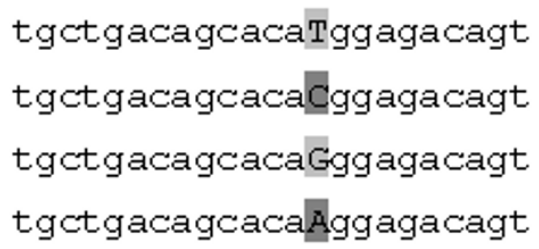

\title{
Figure I
}

Design a 23 mer oligonucleotide for SNP detection. In (a) the relevant PCR-product of I66 bp is shown. (b) exhibits the set of oligonucleotides ( 12 for sense and 12 for antisense strand; at $n=0$ the allele is located in the middle of the oligomer, at $\mathrm{n}=-2$ and $\mathrm{n}=+2$ the SNP is shifted by 2 nucleotides to the left and right, respectively.

matches - MM). PM/MM scheme enables in addition subtract directly both the background level and crosshybridization signals providing thus with redundancy required for the reliable microarray analysis. The perfect match probe (PM) is designed complementary to the target sequence and the so-called mismatch probe (MM) is identical with the PM, except the base in the middle of the sequence. Ideally, there is 30 -fold difference in the signal intensities of PM vs. MM oligo. In hybridization the oligo signal intensity depends directly of its sequence GC content. Depending on sequence content (high G/C content) the $\mathrm{MM}$ oligo can result sufficiently high signal and interfere discrimination between PM and MM signals. In such cases the entire set of 24 oligoprimers, specially designed for detection of one SNP from sense and antisense strands, is underperforming and has to be left out of array design. In addition, we tested positional effects by moving the polymorphic nucleotide from the center to positions +2 and -2 as well as +1 and -1 of the oligonucleotide probes (Fig. 1). This shift resulted in differences in signal intensities but did not add to the overall amount of information that could be gathered from an experiment. In consequence, we decided to use only probes that contained the respective SNP in a central position but placed three copies of the same oligosequence at different locations of the microarray. 
Table I: Relative allele frequences of SNPs genotyped in 19 hay fever patients with extreme IgE phenotype and I9 non-atopic controls.

\begin{tabular}{|c|c|c|c|c|c|}
\hline SNP Nr. & SNP name & SNP identifier & Allele I & $\begin{array}{c}\text { Relative frequence } \\
\text { of allele } I \text { in case } \\
\text { sample }\end{array}$ & $\begin{array}{c}\text { Relative frequence } \\
\text { of allele } I \text { in } \\
\text { control sample }\end{array}$ \\
\hline I & IL-2_I & rs2069772 & $\mathrm{T}$ & 0.58 & 0.83 \\
\hline 2 & IL-2_2 & rs2069763 & G & 0.67 & 0.61 \\
\hline 3 & IL-10_4 & rsl800894 & $\mathrm{G}$ & 1.00 & 1.00 \\
\hline 4 & IL-10_5 & rs|80087I & $\mathrm{C}$ & 0.77 & 0.83 \\
\hline 5 & IL-10_6 & rs|800872 & $\mathrm{C}$ & 0.77 & 0.83 \\
\hline 6 & TNFA_7 & rslI565 & $\mathrm{C}$ & 0.85 & 0.92 \\
\hline 7 & TNFA_8 & rs673 & $A$ & 0.03 & 0.03 \\
\hline 8 & TNFA_9 & rs|800629 & $A$ & 0.16 & 0.05 \\
\hline 9 & TNFA_10 & rs361525 & A & 0.11 & 0.03 \\
\hline 10 & IL4_II & rs2243246 & $\mathrm{T}$ & 0.95 & 0.86 \\
\hline 11 & IL4_I2 & rs2243250 & C & 1.00 & 1.00 \\
\hline 12 & IL4_I3 & rs34185442 & $\mathrm{C}$ & 1.00 & 1.00 \\
\hline 13 & IL4_I4 & rs2970874 & $\mathrm{C}$ & 1.00 & 0.97 \\
\hline 14 & IL6_16 & rsl800797 & G & 0.63 & 0.60 \\
\hline 15 & IL6_I6 & rsl800796 & G & 1.00 & 1.00 \\
\hline 16 & IL6_I7 & rs|800795 & G & 0.63 & 0.47 \\
\hline 17 & IL4R_I8 & rs|80I275 & $A$ & 0.87 & 0.68 \\
\hline 18 & IL4R_19 & rsl8050II & C & 0.05 & 0.13 \\
\hline 19 & IL4R_20 & rs8832 & $\mathrm{G}$ & 0.75 & 0.62 \\
\hline 20 & IL4R_2I & rs 1805015 & $\mathrm{~T}$ & 1.00 & 0.89 \\
\hline 21 & IL4R_22 & rs 1805010 & $A$ & 0.70 & 0.56 \\
\hline 22 & ILI $2 p 40 \_23$ & $r s 3124$ & C & 1.00 & 1.00 \\
\hline 23 & STAT6_24 & rs167769 & $A$ & 0.32 & 0.20 \\
\hline 24 & STAT6_25 & rs3240I5 & G & 0.72 & 0.82 \\
\hline 25 & STAT6_26 & rs7038I7 & $\mathrm{G}$ & 0.50 & 0.38 \\
\hline $26^{*}$ & STAT6_27 & rs4559 & A & 0.27 & 0.25 \\
\hline 27 & IFNG_28 & rs2234685 & A & 1.00 & 1.00 \\
\hline 28 & IFNG_29 & rs|86|493 & $\mathrm{T}$ & 0.69 & 0.69 \\
\hline 29 & IFNG_30 & rs2234687 & C & 1.00 & 1.00 \\
\hline $30 *$ & IFNG_3I & rs243056I & $\mathrm{T}$ & 0.50 & 0.38 \\
\hline 31 & IFNGR2_34 & rsl802585 & C & 1.00 & 1.00 \\
\hline 32 & IFNGR2_35 & rsl059293 & $\mathrm{T}$ & 0.39 & 0.50 \\
\hline 33 & IFNGR2_36 & rs9808753 & $A$ & 0.86 & 0.94 \\
\hline 34 & IRFI_37 & rs839 & G & 0.74 & 0.89 \\
\hline $35^{*}$ & IRFI_38 & rs9282762 & $A$ & 0.42 & 0.60 \\
\hline $36^{*}$ & IRF2_40 & rsII3I553 & G & 0.32 & 0.50 \\
\hline 37 & IL8_4I & rslli75 & $A$ & 0.41 & 0.47 \\
\hline 38 & IL8_42 & rs2227307 & G & 0.42 & 0.50 \\
\hline 39 & ILI $3 \_43$ & rs2054I & G & 0.89 & 0.79 \\
\hline 40 & ILI3_44 & rs 1800925 & C & 0.88 & 0.82 \\
\hline $41^{*}$ & ILI8_47 & rs19465I8 & $\mathrm{G}$ & 0.63 & 0.67 \\
\hline $42^{*}$ & ILI8_48 & rs 1946519 & C & 0.87 & 0.71 \\
\hline $43^{*}$ & ILIB_49 & rsl6944 & $\mathrm{T}$ & 0.16 & 0.15 \\
\hline 44 & ILIB_50 & rsl|43627 & $\mathrm{C}$ & 0.36 & 0.38 \\
\hline 45 & |LIB_5I & rs|7999|6 & $\mathrm{T}$ & 1.00 & 1.00 \\
\hline 46 & ILIA_52 & rsl756I & G & 0.64 & 0.74 \\
\hline 47 & ILIA_53 & rs 1800587 & $\mathrm{~T}$ & 0.36 & 0.31 \\
\hline 48 & IL9_56 & rsl799962 & A & 1.00 & 1.00 \\
\hline 49 & TNFRI_60 & rsl800692 & $\mathrm{C}$ & 0.63 & 0.53 \\
\hline 50 & TNFRI_6I & rsl800693 & A & 0.53 & 0.72 \\
\hline $51^{*}$ & TNFRSF6_62 & rs2234768 & $\mathrm{T}$ & 0.00 & 1.00 \\
\hline 52 & LTA_65 & rs|800683 & A & 1.00 & 1.00 \\
\hline 53 & LTA_66 & rsl041981 & A & 0.29 & 0.19 \\
\hline 54 & LTA_67 & rs909253 & G & 0.25 & 0.19 \\
\hline 55 & ILIRN_68 & rs2234676 & $\mathrm{G}$ & 0.86 & 0.74 \\
\hline 56 & ILIRN_69 & rs419598 & $\mathrm{T}$ & 0.87 & 0.88 \\
\hline 57 & CTLA4_70 & rs2384I37 & G & 0.11 & 0.06 \\
\hline
\end{tabular}


Table I: Relative allele frequences of SNPs genotyped in 19 hay fever patients with extreme IgE phenotype and I 9 non-atopic controls. (Continued)

\begin{tabular}{|c|c|c|c|c|c|}
\hline 58 & NFKBIA_72 & rs 1800439 & $\mathrm{G}$ & 0.53 & 0.56 \\
\hline 59 & IL8RB_77 & rs2230054 & $\mathrm{T}$ & 0.06 & 0.17 \\
\hline 60 & ICAMI_78 & rsI799969 & $A$ & 1.00 & 0.97 \\
\hline $61^{*}$ & ICAMI_79 & rs5498 & G & 0.61 & 0.64 \\
\hline 62 & IL3_8I & rs4040I & $\mathrm{G}$ & 0.95 & 0.94 \\
\hline 63 & IL3_82 & rs31480 & $\mathrm{G}$ & 0.84 & 0.82 \\
\hline 64 & MCPI_87 & rs46II5II & $A$ & 0.89 & 0.92 \\
\hline 65 & MCPI_88 & rs34020694 & $A$ & 0.87 & 0.87 \\
\hline 66 & RANTES_89 & rs2107538 & $\mathrm{G}$ & 0.88 & 0.91 \\
\hline 67 & RANTES_90 & rs2280788 & C & 0.95 & 0.95 \\
\hline 68 & CCR5_9l & rsI799863 & $A$ & 0.03 & 0.00 \\
\hline 69 & CCR2_94 & rs|799865 & $\mathrm{T}$ & 0.50 & 0.56 \\
\hline 70 & C5_95 & rsI76II & G & 0.66 & 0.50 \\
\hline 71 & C5_96 & rs 17612 & $\mathrm{C}$ & 0.11 & 0.06 \\
\hline 72 & P2X7_97 & rs 3751143 & $\mathrm{C}$ & 0.06 & 0.03 \\
\hline 73 & IL7R_I06 & rs|494555 & G & 0.34 & 0.35 \\
\hline 74 & PRFI_I07 & rs885822 & $\mathrm{T}$ & 0.83 & 0.94 \\
\hline 75 & TLR2_108 & rsI804965 & G & 1.00 & 1.00 \\
\hline 76 & TCLIB__109 & rs1064017 & $\mathrm{G}$ & 0.44 & 0.56 \\
\hline 77 & CCR5_IIO & rs 1800452 & $\mathrm{G}$ & 1.00 & 1.00 \\
\hline 78 & ILII_III & rsII26757 & $A$ & 0.38 & 0.44 \\
\hline 79 & ILII_II & rs2298885 & G & 0.61 & 0.85 \\
\hline 80 & IL8RA_II7 & rs223467I & $\mathrm{G}$ & 0.40 & 0.44 \\
\hline $81^{*}$ & ILILI_II8 & rs 1800930 & $A$ & 0.78 & 0.83 \\
\hline $82^{*}$ & CD36_II9 & rs 1334512 & G & 0.96 & 0.85 \\
\hline 83 & VDR_I2I & rsI5444I0 & $\mathrm{G}$ & 0.31 & 0.44 \\
\hline $84^{*}$ & VDR_122 & rs7975232 & $\mathrm{T}$ & 0.73 & 0.54 \\
\hline 85 & IL5RA_I23 & rs 2290610 & $A$ & 0.83 & 0.61 \\
\hline 86 & IL5R_I24 & rs 2069812 & C & 0.79 & 0.78 \\
\hline 87 & IL5R_I25 & rs20698I8 & $\mathrm{C}$ & 1.00 & 1.00 \\
\hline 88 & CX3CRI_I26 & rs3732379 & $\mathrm{G}$ & 0.68 & 0.67 \\
\hline 89 & CX3CRI_I 27 & rs3732378 & C & 0.78 & 0.74 \\
\hline $90 *$ & TNFRSFIB_I 128 & rs 1061622 & $\mathrm{~T}$ & 0.88 & 0.65 \\
\hline 91 & TNFRSFIB_I29 & rs1061624 & $A$ & 0.31 & 0.47 \\
\hline 92 & TNFRSFIB_I 30 & rs3397 & $\mathrm{T}$ & 0.88 & 0.79 \\
\hline $93 *$ & TNFRSFIA_I3I & rs887477 & G & 0.54 & 0.35 \\
\hline 94 & TNFRSFIA_I 32 & rs4l49570 & $\mathrm{G}$ & 0.76 & 0.53 \\
\hline 95 & IL4R_I 35 & rs 1805016 & $\mathrm{~T}$ & 1.00 & 1.00 \\
\hline 96 & IL6_137 & rs 20069860 & $A$ & 0.97 & 1.00 \\
\hline $97 *$ & IL9_I38 & rs20069885 & C & 1.00 & 0.96 \\
\hline $98^{*}$ & NKFB_139 & rs1020759 & $\mathrm{C}$ & 1.00 & 1.00 \\
\hline 99 & GATA3_I4I & rs57013 & $A$ & 0.72 & 0.58 \\
\hline
\end{tabular}

* The SNP detection reproducibility $<80 \%$

Furthermore, different temperatures for hybridisation $\left(40^{\circ} \mathrm{C}, 45^{\circ} \mathrm{C}, 50^{\circ} \mathrm{C}\right.$, and $\left.55^{\circ} \mathrm{C}\right)$ and changes in hybridisation time from one to four hours were compared. The time of hybridisation in this experiment had little influence on number of correct and false signals. However, increased hybridisation temperature at $50^{\circ} \mathrm{C}$ or $55^{\circ} \mathrm{C}$ reduced cross hybridisation at least $5 \%$ and lowered general amount of positive signal to $60 \%$ and $40 \%$ (respectively). Reduced stringency by decreased hybridisation temperature maximized the overall number and intensity of signals, but this was accompanied with $30 \%$ increase of unspecific hybridisation signals.
We also varied probe length, synthesizing on the same chip oligonucleotides of 19, 21, 23, 25 and 27 nucleotides. While longer sequences usually produce higher signal intensities, shorter oligonucleotides permit better discrimination of single base differences due to the more pronounced destabilizing effect of a mismatch. As expected, the signal intensities of both the fully matched $\left(I_{1}\right)$ and the mismatch probes $\left(I_{2}\right)$ increased with length while discrimination $\left(\mathrm{I}_{1} / \mathrm{I}_{2}\right)$ improved the shorter the oligonucleotides were (Fig. 2). Measured signal intensity $\left(\mathrm{I}_{1}\right)$ increases clearly with higher nucleotides number in the sequence of oligonucleotide-probe: $\mathrm{I}_{1}(27 \mathrm{bp})>\mathrm{I}_{1}(19 \mathrm{bp})$ (Fig. 3). Same effect is obtained for $\mathrm{MM}\left(\mathrm{I}_{2}\right)$ oligo-probes as well. Though the discrimination between PM/MM 


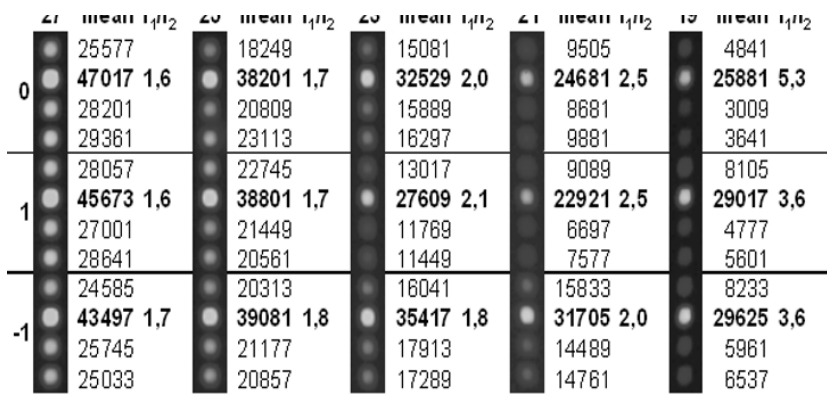

Figure 2

The dependence of signal intensity on oligonucleotide length. Hybridization was done at $45^{\circ} \mathrm{C}$. $l_{1} / I_{2}$ labels the ratio of the signal at the full-match oligonucleotide and the signals at the mismatched oligonucleotides. 27, 25, 23, 2 I and 19 indicates the length of oligomers. The SNP was located either at the center of the oligonucleotides $(0)$ or shifted by two bases in either direction $(+I,-I)$.

according to the calculated relation of measured intensities $\left(I_{1} / I_{2}\right)$ is higher for shortest set of oligo-probes as 19 bp $(5,3 \ldots 3,6)$ and the lowest with 27 bp $(1,7 \ldots 1,6)$ ones. Variation of $I_{1} / I_{2}$ among probes within the same number of nucleotides comes mainly from GC content differences/variations of probe-sequence itself. Why longer $\mathrm{MM}$ sequences give higher signal comparing to shorter ones comes mainly from weaker destabilizing effect of noncomplimentary nucleotide on formation of doublestranded complex between probe and target DNA.

Tests at different hybridization temperatures $\left(40-55^{\circ} \mathrm{C}\right)$ produced the overall best results for the majority of SNPs with 23-mer probes and 3 to 4 hours of hybridization at $45^{\circ} \mathrm{C}$. Finally, the selected set of oligoprobes, as well as the hybridization conditions, were tested in addition with 4 genomic DNA samples of control individuals. These control experiments had 5-fold redundancy. Concordance of analyzed genotypes were compared individually.

For selecting the best performing oligoprobes in the initial optimization experiments one test-DNA with good quality was used. All hybridization reactions from chip design step were repeated 3 times. During the optimization process, we identified several oligonucleotide probes that did not perform irrespective of the chosen hybridization conditions (e.g., Fig. 2). Apparently, the previously described selection basis of cross-reactivity could be even more
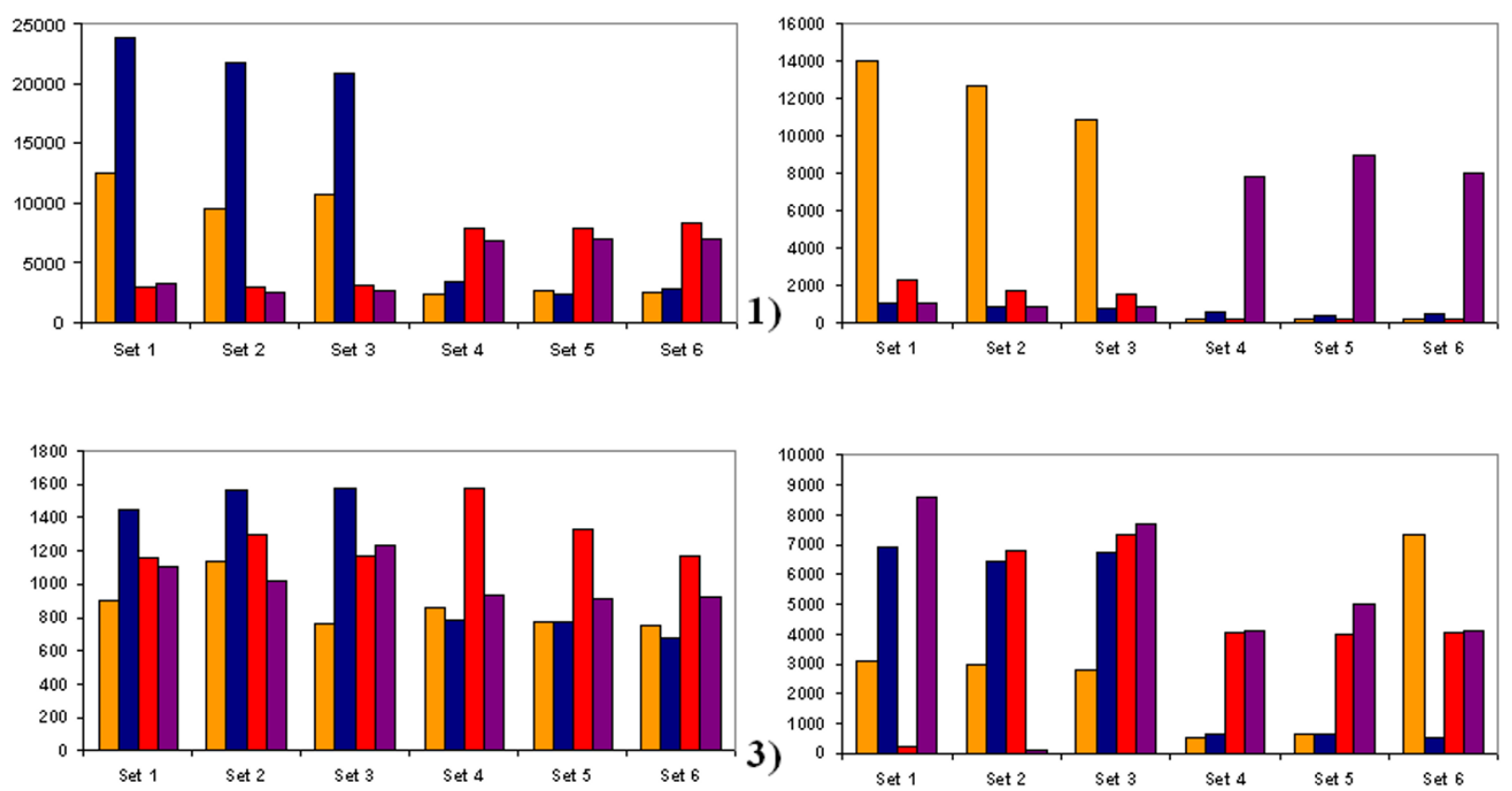

Figure 3

Differences in the performance of oligonucleotides. Set I to 6 label the oligonucleotides designed for detecting a SNP. Each column indicates the signal intensity at the oligomers that represent (left to right) the A, G, C or T variant of a sequence. Sets I to 3 are the data produced on replicate microarray positions that represent one strand, while sets 4 to 6 indicate the signal intensities produced by the complementary DNA strand. Panel (I) shows the result obtained for a heterozygous sample, panel (2) a homozygous sample. Panel (3) exhibits data obtained with an oligonucleotide that was predicted in silico to perform well but failed in the experiment. In panel (4) a result of an oligomer with a high degree of cross-hybridization is presented. 


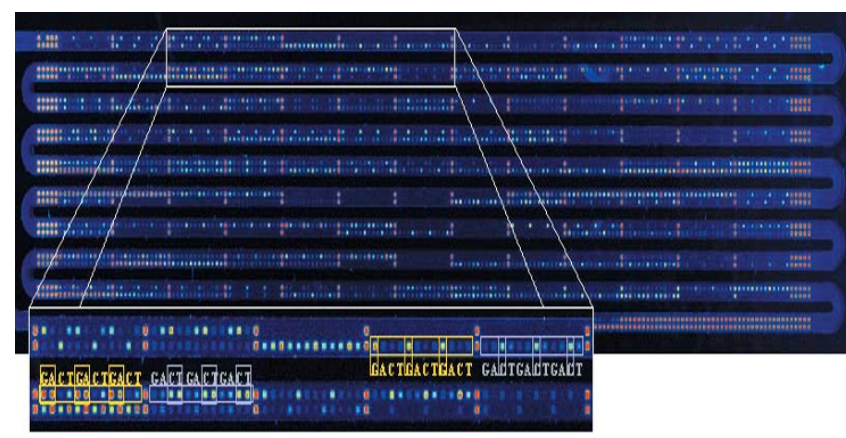

Figure 5

Image of a simultaneous hybridization of 99 PCRproducts to an in situ synthesized oligonucleotide microarray. Usually, the features were scrambled across the array. For illustrative purposes, they were placed next to each other in this particular experiment.

stringent e.g. we should allow less base pairing. Following experimental tests revealed additional oligoprobe sets falling out from final chip design because of the same reason. Herewith, basis on experimental results, the threshold for software based oligo probe selection could be set on $80 \%$ allowing less base pairing (and less false signals due to nonspecific oligo hybridization) than $85 \%$. In total, 29 out of the 128 SNPs (22\%) could not be analyzed adequately. The respective oligomer probes that had been defined as good by the in silico selection process were empirically found to be ill-performing in real hybridizations. Either the absolute signal intensity was too low to permit a statistically solid analysis or the discriminative effect was insufficient. The ratio between PM and MM oligo signal intensities is supposed to be at least $1 / 3$ (Fig. 3 ). The high number of failing oligonucleotides illustrates the need for a careful experimental validation of in silico designed microarrays.

Using the optimized microarray, we performed genotyping analyses at 99 SNPs in 68 genes that have a putative functional significance for the occurrence of hay-fever. From a case-control study on hay-fever [10], 19 cases with the most extreme plasma IgE levels against inhalant antigens and 19 age- and sex-matched non-atopic controls were selected. Informed consent of the participants was given in writing and the local ethics committee approved the study. PCR-amplifications of the relevant DNAregions were performed either individually or in pools of 5 or 10 samples. While all pentaplex reactions yielded a product for each individual band, two decaplex amplifications failed to produce 1 out of the expected 10 amplicons (Fig. 4). The 99 products were pooled prior to labelling and hybridized concomitantly (Fig. 5). For each sample, analysis was repeated up to four times. The observed allele frequencies are presented in Table 1. To assess the accuracy of the genotyping, ten PCR-products of heterozygote calls obtained from the microarray analyses were subjected to gel-based DNA sequencing for confirmation. In all cases, the results were in full agreement.

Hybridization experiments for all studied 38 individuals were repeated twice.

$16 \%$ of SNPs presented only one allele in the 38 studied samples. For 14 samples ( 7 cases and 7 controls) the call rate for all variants was above $90 \%$. And in one case it was below $80 \%$ due to the low quality of this particular DNA sample. For 17 SNPs the amplification step basically failed due to low quality of clinical genomic DNA samples. After exclusion of these particular 17 SNPs (indicated with an asterisk in Tab. 1) that performed poorly in hybridizations the average concordance was $93 \%$. From the variants with high quality data, five SNPs in the genes IL2 (rs2069772), TCL1B (rs1064017), IL11 (rs2298885), IL5RA (rs2290610) and TNFRSF1A (rs4149570) had pvalues smaller than 0.05 for the association of carrying the mutant allele with the high IgE phenotype. The homozygous genotype A for the IL5 receptor alpha (IL5RA Ile129 Val) was associated with a 6.8 -fold risk (95\% confidence interval, 1.6-29.1) of a high IgE phenotype.

\section{Discussion}

An oligonucleotide microarray was produced using GeniomOne device to facilitate the screening of single nucleotide polymorphisms in several genes that are associated with hay-fever as a pilot project. Based on an in silico design, the selected set of oligonucleotides was optimized by a subsequent experimental analysis. While the in silico process eliminated $9 \%$ of the initially 141 SNPs that had been picked purely on the basis of a potential association with the occurrence of hay-fever, the subsequent experimental validation eliminated another $20 \%$ of these oligomers, more than twice as many. This result illustrates the importance of experimental validation of the microarray designs. Even in analyses that are based on a continuous detection of the hybridization and dissociation process (dynamic allele-specific hybridization) [18] the selection is critical, although an analysis of the association and dissociation curves of the duplexes permit a more discriminative and accurate SNP detection.

The reasons for the failing probes could be manifold [19]. Although only short fragments were hybridized, secondary structures formed either within one sample molecule or between different targets could cause inefficient binding to the array-bound probe molecules. Also, it is well known that dangling ends of the target molecules may have a profound effect on the hybridization [20]. Documentation of the effectiveness of the genotyping ability of 


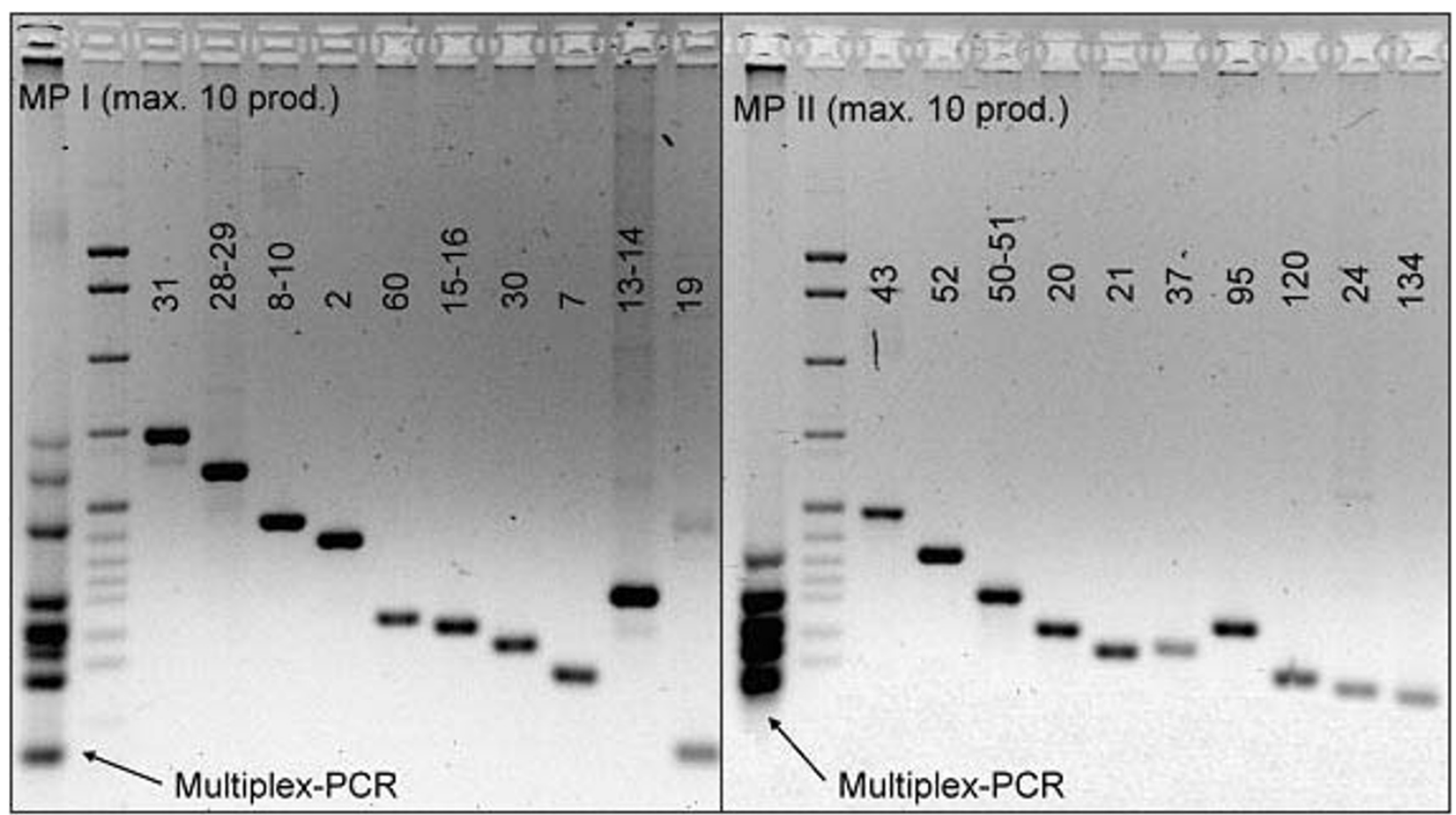

Figure 4

Gel-electrophoretic separation of the products of multiplex-PCR. Two decaplex amplifications are shown in comparison to the respective individual reactions. In both cases presented here, one product was not amplified in the multiplex reaction while the reaction worked fine in the individual amplification.

particular sets of oligonucleotide probes is essential for a study of high accuracy. Use of flexible in situ synthesized oligonucleotide microarrays to such ends appears to be an efficient and attractive method for fast and cost-efficient pre-screening of candidate SNPs for an eventual highthroughput genotyping.

GeniomOne allows synthesizing $8 \times 8.000$ probes per array overnight and test them right after in hybridization experiments. In this way many combinations can be tested in parallel without additional cost, which allows selecting an optimal set of oligoprobes for the following experiments. This is a big advantage of GeniomOne technology.

In the analysis of the 38 DNA samples of hay-fever cases and controls, we were able to identify at least five polymorphisms in immune regulator genes that contribute to the extreme IgE phenotype and deserve further testing. For $22 \%$ of the selected SNPs, only one genotype was seen in 38 individuals. For several variants, allele-frequency differences between cases and controls exceeded 10\%. These include non-synonymous variants in the IL5 receptor alpha (IL5RA Ile129 Val) and TCL1B (Gly93Arg), pro- moter polymorphisms in IL2 (-330 T/G) and TNFRSF1A $(-609 \mathrm{G} / \mathrm{T})$, and a polymorphism in the 3' UTR of IL11. IL5RA is a crucial factor in IL5 signalling and a contributor to the genetics of atopy in mice [21]. The extreme phenotype design of the study performed here may be an efficient alternative for the identification of disease-relevant sequence variants.

\section{Conclusion}

Based on a platform that permits flexible in situ oligonucleotide synthesis, a set of optimally performing probes could be defined by a selection approach that combined computational and experimental aspects. The final design achieved by this process permitted an effective discrimination of both homo- and heterozygote polymorphisms in hay-fever patients. Allele-frequencies of more than $10 \%$ could be identified.

\section{Methods}

\section{Microarray synthesis}

All analysis steps, (i) in situ synthesis of the oligonucleotide microarray, (ii) hybridization of the labeled PCRproduct mixture and (iii) detection of the signal intensities were performed with the GeniomOne device of febit 
Table 2: Primer sequences used for PCR-amplification of the SNP-regions.

\begin{tabular}{|c|c|c|c|c|}
\hline No. & SNP Name & SNP ID & Forward Primer (5'-3') & Reverse Primer (5'-3') \\
\hline I & IL-2_I & rs2069772 & CCATTCTGAAACAGGAAACCA & CTTTAAGGGGGTGGGGATAC \\
\hline 2 & IL-2_2 & rs2069763 & TGCAACTCCTGTCTTGCATT & ACTTACATTAATTCCATTCAAAATCA \\
\hline 3 & IL-10_4 & rs|800894 & TCCAGCCACAGAAGCTTACA & GTGCTCACCATGACCCCTAC \\
\hline 4 & IL-10_5 & rs $180087 \mid$ & TCCAGCCACAGAAGCTTACA & GTGCTCACCATGACCCCTAC \\
\hline 5 & IL- $10 \_6$ & rs 1800872 & TCCAGCCACAGAAGCTTACA & GTGCTCACCATGACCCCTAC \\
\hline 6 & TNFA_7 & rsII565 & ACCACAGCAATGGGTAGGAG & CATGCCCCTCAAAACCTAT \\
\hline 7 & TNFA_8 & rs 673 & ACCACAGCAATGGGTAGGAG & CGTCCCCTGTATTCCATACCT \\
\hline 8 & TNFA_9 & rs 1800629 & GCCCCTCCCAGTTCTAGTTC & GCATCAAGGATACCCCTCA \\
\hline 9 & TNFA_10 & rs361525 & GCCCCTCCCAGTTCTAGTTC & GCATCAAGGATACCCCTCA \\
\hline 10 & IL4_II & rs2243246 & GCCCCTCCCAGTTCTAGTTC & GCATCAAGGATACCCCTCA \\
\hline 11 & IL4_I2 & rs2243250 & AGTGAGTGGTGGGGTCCTTA & AATGCCCACTTTTTTGAATGG \\
\hline 12 & IL4_I3 & rs34185442 & ACCCAAACTAGGCCTCACCT & GGTGGCATCTTGGAAACTGT \\
\hline 13 & IL4_I4 & rs2970874 & GGAAGAGAGGTGCTGATTGG & CGATTTTGCAGTGACAATGTG \\
\hline 14 & IL6_I6 & rs 1800797 & GGAAGAGAGGTGCTGATTGG & CGATTTGCAGTGACAATGTG \\
\hline 15 & IL6_16 & rs 1800796 & TGGCAAAAAGGAGTCACACA & CCCAAGCCTGGGATTATGAAG \\
\hline 16 & IL6_17 & rs 1800795 & TGGCAAAAAGGAGTCACACA & CCCAAGCCTGGGATTATGAAG \\
\hline 17 & IL4R_I8 & rs $180 \mid 275$ & GCTAGCCTCAATGACGACCT & TCATGGGAAAATCCCACATT \\
\hline 18 & IL4R_19 & rsl8050II & GAAACCTGGGAGCAGATCCTC & GGCCTTGTAACCAGCCTCTC \\
\hline 19 & IL4R_20 & rs8832 & AAAGGGAGCTTCTGTGCATC & TCTCCGAGCTGGTCCAG \\
\hline 20 & IL4R_2I & rs 1805015 & TTCCTTAGGTTGATGCTGGAG & GGTTCCATGCATACGAGGAG \\
\hline 21 & IL4R_22 & rs 1805010 & ACCTGACTTGCACAGAGACG & AGGGCATGTGGGTTCTACT \\
\hline 22 & ILI2p40_23 & rs 3124 & GCCTACAGGTGACCAGCCTA & AGCCCACGGTCCAGTGTAT \\
\hline 23 & STAT6_24 & rs 167769 & CACAACGGAATAGACCCAAAA & ATGGCAACTTGAGAGCTGGA \\
\hline 24 & STAT6_25 & rs3240I5 & GCACTGACTGGAAGGGAAGT & СССТAACCTGTGCTCTTACCC \\
\hline 25 & STAT6_26 & rs7038I7 & GTCTCAGCCCTAGGGGAATG & CTCCACCTGGCTAACAGGAA \\
\hline 26 & STAT6_27 & rs 4559 & CAAAAGTACAAGGGCTGA & CCCAAATTTGTGTTGTCACG \\
\hline 27 & IFNG_28 & rs2234685 & GGAAGTAGGTGAGGAAGAAGCG & TGGAGCAAAGAAGGTCATCA \\
\hline 28 & IFNG_29 & rs 1861493 & GGAAGTAGGTGAGGAAGAAGCG & TGGAGCAAAGAAGGTCATCA \\
\hline 29 & IFNG_30 & rs2234687 & TCCCATGGGTTGTGTGTTTA & GGGTCACCTGACACATTCAA \\
\hline 30 & IFNG_31 & rs243056I & TTCAGACATTCACAATTGATTTTTATTC & CCCCAATGGTACAGGTTTCT \\
\hline 31 & IFNGR2_34 & rs 1802585 & CCCAACTCAGCCCATCTTAG & ATCTCTTCCAGGGAGCCAGT \\
\hline 32 & IFNGR2_35 & rs 1059293 & GGGCTGAGCAGTCAGAA & CATTTTAAGCCAGCACACCA \\
\hline 33 & IFNGR2_36 & rs9808753 & CAGAGCAGGTCCTGAGTTGGGAGC & GTTTCCCACGGGTTTGATAA \\
\hline 34 & IRFI_37 & rs839 & GGACTGTTCCAAAGCCAGTG & CAGAAATGTGGCAAGATCCA \\
\hline 35 & IRFI_38 & rs9282762 & TTGCAAACTAAGAAAGCACACAA & ATGGGTGACACCTGGAAGTT \\
\hline 36 & IRF2_40 & rsl|3|553 & CTCCCAAAGTGCTGGGATTA & CTGTTGTAAGGCACCGGATT \\
\hline 37 & IL8_4I & rsll175 & СTTCACCATCATGATAGCATCTGT & GGAGTATGACGAAAGTTTTCTTTG \\
\hline 38 & IL8_42 & rs2227307 & TGCTTTGGTAACAAACATCCTTT & GGTAACCGTCCTTCTCAATAGG \\
\hline 39 & ILI3_43 & rs2054l & CTTCCGTGAGGACTGAATGAGAC & CTGCAAATAATGATGCTTTCGAAGTTTCAG \\
\hline 40 & ILI3_44 & rs|800925 & TGACATCAACACCCAACAGG & GCAGAATGAGTGCTGCTGTGGAG \\
\hline 41 & ILI8_47 & rs 1946518 & GGTCAGTCTTTGCTATCATTCCAG & AGCCACACGGATACCATCAT \\
\hline 42 & ILI8_48 & rs 1946519 & GGTCAGTCTTTGCTATCATTCCAG & AGCCACACGGATACCATCAT \\
\hline 43 & ILIB_49 & rs 16944 & AGCCTGAACCCTGCATACC & CAATAGCCCATCCCTGTCTGT \\
\hline 44 & ILIB_50 & rs II43627 & TCTCAGCCTCCTACTTCTGCTT & CAGAGAGACTCCCTTAGCACCT \\
\hline 45 & ILIB_5I & rs1799916 & TCTCAGCCTCCTACTTCTGCTT & CAGAGAGACTCCCTTAGCACCT \\
\hline 46 & ILIA_52 & rs 17561 & СССССТCCAGAACTATTTTCC & ACTTTGATTGAGGGCGTCAT \\
\hline 47 & ILIA_53 & rs 1800587 & TAGGCTGGCCACAGGAATTA & AGCCAGAACCAGTGGCTAAG \\
\hline 48 & IL9_56 & rs 1799962 & CCTTCGTTAGAACACCCATGA & AGACAGGGATTCTGGTGTGA \\
\hline 49 & TNFRI_60 & rs 1800692 & TCCCCCTCCTGTATTCTGTG & GTGCACACGGTGTTCTGTTT \\
\hline 50 & TNFRI_6I & rsl800693 & CCTGGAGTGCACGAAGTTGT & ATAGATGGATGGGTGGGATG \\
\hline 51 & TNFRSF6_62 & rs2234768 & САТССТССТТАТСССАСТТСТTТ & CACCCTGTGTTTTGCATCTA \\
\hline 52 & LTA_65 & rs 1800683 & САCTGCCGCTTCCTCTATAA & GGTAGTCCAAAGCACGAAGC \\
\hline 53 & LTA_66 & rs 1041981 & СССССТСААСТСТGТTСТСС & GGGAGGTCAGGTGGATGTTT \\
\hline 54 & LTA_67 & rs909253 & GGGTTTGGTTTTTGGTTTTCCT & CAGAGAAACCCCAAGGTGAG \\
\hline 55 & ILIRN_68 & rs2234676 & GCCCATCTCCTCATGCTG & GCTGCTGCCCATAAAGTAG \\
\hline 56 & ILIRN_69 & rs419598 & TCCTTTTCAGAATCTGGGATGT & CGTGATGCCCAGATACATTG \\
\hline 57 & CTLA4_70 & rs2384I37 & AACACCGCTCCCATAAAGC & ССТССТССАТСТТСАТGСТС \\
\hline 58 & NFKBIA_72 & rs 1800439 & СCTTGTTTTCAGCTGCCCTA & TCGTCCCCTACAAAAAGTTCA \\
\hline 59 & IL8RB_77 & rs2230054 & ACATTCCAAGCCTCATGTCC & TACCAGGGCAGGCTTTCTA \\
\hline 60 & ICAMI_78 & rsl799969 & CTTGAGGGCACCTACCTCTG & AGGATACAACAGGCGGTGAG \\
\hline 61 & ICAMI_79 & rs5498 & CTTGAGGGCACCTACCTCTG & AGGATACAACAGGCGGTGAG \\
\hline 62 & IL3_8I & rs 40401 & GAGCAGTTAACCCAGCTTGTC & CACCTTGCTGCTGCACATA \\
\hline 63 & IL3_82 & $r s 31480$ & GAGCAGTTAACCCAGCTTGTC & CACCTTGCTGCTGCACATA \\
\hline 64 & MCPI_87 & rs 4611511 & AAAGCTGCCTCCTCAGAGTG & CACAGGGAAGGTGAAGGGTA \\
\hline 65 & MCPI_88 & rs34020694 & AGAGAAAACCCGAAGCATGA & TCTTCCTAGGCCATCTCACC \\
\hline 66 & RANTES_89 & rs2107538 & ATCCAGAGGACCCTCCTCAA & GGAGTGGCAGTTAGGACAGG \\
\hline 67 & RANTES_90 & rs2280788 & TTCTTTTCCGTTTTGTGCAAT & CGTGCTGTCTTGATCCTCTG \\
\hline 68 & CCR5_9l & rsl799863 & CTGCCTCCGCTCTACTCACT & GCCAGGTTGAGCAGGTAG \\
\hline
\end{tabular}


Table 2: Primer sequences used for PCR-amplification of the SNP-regions. (Continued)

\begin{tabular}{|c|c|c|c|c|}
\hline 69 & CCR2_94 & rs I799865 & AGAGGCATAGGGCAGTGAGA & GGTCCAGTTGACTGGGTGCTT \\
\hline 70 & C5_95 & rs 1761I & TGCAGTTTGCCCTACCTGAT & TGCTACCATTTAAGTCCTGGGTA \\
\hline 71 & C5_96 & rs 17612 & TTTTAGCTACAAGCCCAGCA & AATGAAGCATTCACAACACGA \\
\hline 72 & P2X7_97 & rs375II43 & TTCCTGGACAACCAGAGGAG & ACCAGCTTCCTGAACAGCTC \\
\hline 73 & IL7R_I06 & rs 1494555 & CACTATAGTTAAACCTGAGGCTCC & TCCTGGCGGTAAGCTACATC \\
\hline 74 & PRFI_107 & rs885822 & CCCAGGTCAACATAGGCATCC & CGAACAGCAGGTCGTTAAT \\
\hline 75 & TLR2_108 & rs 1804965 & ATTCTTCTGGAGCCCATTGA & GGACTTTATCGCAGCTCTCA \\
\hline 76 & TCLIB_109 & rs 1064017 & ACAGTGCACTTGTGGCAG & CTGGCCATGGTCTGCTATTT \\
\hline 77 & CCR5_IIO & rs 1800452 & TAGTCATCTTGGGGCTGGTC & TGTAGGGAGCCCAGAAGAGA \\
\hline 78 & ILIIIIII & rs I I 26757 & GGGACCACAACCTGGATTC & ATCAGAGAACACCCGACCAG \\
\hline 79 & ILIIIIII2 & rs2298885 & GGCTGTGTTCACCATAGCAA & ATCCCAAGCAAGCCTCTCTC \\
\hline 80 & IL8RA_II7 & rs223467I & CATCTTTGCTGTCGTCCTCA & CCAGAATCTCAGTGGCATCC \\
\hline 81 & ILILI_II8 & rs 1800930 & GATGGTGCTACTGCTGTGGA & GGGCTCAGGGTAACACTG \\
\hline 82 & CD36_119 & $r s \mid 334512$ & CTGGCAACAAACCACACACT & TCCTACACTGCAGTCC \\
\hline 83 & VDR_I2I & rs 1544410 & ССTCACTGCCCTTAGCTCTG & CAGGAATGTTGAGCCCAGTT \\
\hline 84 & VDR_I22 & rs7975232 & CTGCCGTTGAGTGTCTGTGT & ACGTCTGCAGTGTGTTGGAC \\
\hline 85 & IL5RA_I23 & rs2290610 & CCATGGCAATGTTTTGTCCT & CAGGTGCAGTGAAGGGAAAC \\
\hline 86 & IL5R_I24 & rs2069812 & СTTGCTTTTTTCCTGCTGCTC & AGTCCAGGAATGGAGGCTCT \\
\hline 87 & IL5R_I25 & rs20698I8 & TGTGGAGAAGAAAGACGGAGA & CAAAATCTTTGGCTGCAACAAACCA \\
\hline 88 & CX3CRI_I26 & rs3732379 & GGTGGTCATCGTGTTTTTCC & AGGCAACAATGGCTAATGC \\
\hline 89 & CX3CRI_I27 & rs3732378 & GGTGGTCATCGTGTTTTTCC & AGGCAACAATGGCTAATGC \\
\hline 90 & TNFRSFIB_I28 & rs 1061622 & СTCCTGACCAAGCCTCCTC & GTCACTGGCTGGGGTAAGTG \\
\hline 91 & TNFRSFIB_I29 & rs 1061624 & TCCTCTAGTGCCCTCCACAG & CACAGAGAGTCAGGGACTTGC \\
\hline 92 & TNFRSFIB_I30 & rs3397 & TCCTCTAGTGCCCTCCACAG & CACAGAGAGTCAGGGACTTGC \\
\hline 93 & TNFRSFIA_I3I & rs887477 & CAGCACAACTGGTCAGAACC & ССТССТСССAGTTCAACAAG \\
\hline 94 & TNFRSFIA_I32 & rs4149570 & TACAGGAACCCCAGGAGACA & TGGGTTCCAATTCAGAATGCTT \\
\hline 95 & IL4R_I35 & rs 1805016 & GTGTCATGGCCAGGAGGAT & AGACTGGCCTCCAGTGGAAC \\
\hline 96 & IL6_137 & rs20069860 & TCССТCСACTGCAAAGGATT & CTGCAGCCACTGGTTCTGT \\
\hline 97 & IL9_I38 & rs20069885 & ACTTTCATCC CCACAGT & TTGCСTCTCATCССТСТСАT \\
\hline 98 & NKFB_139 & rs1020759 & TGCTTCCСTCTTGTGTTTCA & GGGGATGACCTTTAAGTGGA \\
\hline 99 & GATA3_|4| & rs57013 & TCCATCCATTGCACTGAGTC & CCAGAGCAGCTGGTTTAAGTG \\
\hline
\end{tabular}

biotech (Heidelberg, Germany) according to the manufacturer's instructions. The reaction carrier (DNA-processor) represents a microstructured disposable system that consists of four or eight individual arrays, respectively, which can be used individually or in any combination [8]. Controlled by a mask-free, light-controlled process, oligonucleotide probes were synthesized in situ in 3' to 5' direction [9]. For each selected SNP, 24 oligonucleotide probes were synthesized, 12 for either DNA strand (Fig. 1), all designed to exhibit similar hybridization characteristics. The arrays used in this study consisted of 7,448 distinct oligonucleotides (594 perfect match probes and 6,534 mismatch probes, plus 320 copies of a control oligonucleotide). A complete list can be obtained from the authors.

\section{PCR-amplification}

For each SNP, PCR-primers were designed for the amplification of the relevant DNA-fragment using the Primer3 program [22]. All primers have a Tm value of $60^{\circ} \mathrm{C}$. The oligonucleotides were obtained from Thermo Hybaid (Ulm, Germany). Their sequences are presented in the Table 2. The length of the PCR-products varies between $100 \mathrm{bp}$ and $270 \mathrm{bp}$.

Genomic DNA from lymphocytes was extracted using the QiaAmp Blood kit according to the manufacturer's instructions (Qiagen, Hilden, Germany). PCR-amplifications of individual loci were carried out in a Mastercycler gradient thermocycler (Eppendorf-Netheler-Hinz, Hamburg, Germany) in $25 \mu$ lof $1 \times$ Thermoprime polymerase puffer (AB Gene, Epson, UK), $200 \mu \mathrm{mol} / \mathrm{l}$ of each deoxynucleotide triphosphate (Qiagen), $1.5 \mathrm{mmol} / \mathrm{L} \mathrm{MgCl}_{2}(\mathrm{AB}$ Gene), 0.25 units Thermoprime DNA polymerase (AB Gene), 1.0 to $2.5 \mu \mathrm{mol} / \mathrm{l}$ of both forward and reverse primer, and 20 ng DNA. The initial annealing occurred at $94^{\circ} \mathrm{C}$ for $2 \mathrm{~min}$, followed by 30 cycles of $94^{\circ} \mathrm{C}$ for $40 \mathrm{sec}$, $57^{\circ} \mathrm{C}$ for $40 \mathrm{sec}$ and $72^{\circ} \mathrm{C}$ for $30 \mathrm{sec}$. Subsequently, a final extension step was performed at $72^{\circ} \mathrm{C}$ for $1 \mathrm{~min}$.

Multiplex-PCR was performed in a total volume of $25 \mu \mathrm{l}$ solution containing $80 \mathrm{ng}$ human genomic DNA, 1.2 $\mu \mathrm{mol} / \mathrm{l}$ of each primer, $1 \mathrm{mmol} / \mathrm{l}$ deoxynucleotide triphosphates (dNTPs), $5 \mathrm{mmol} / 1 \mathrm{MgCl}_{2}$ and 2 units of Thermoprime Plus DNA polymerase (AB Gene). All primer pairs had been checked in silico for possible primer dimers using the program "Primer Premier 5" (Premier Biosoft International, Palo Alto, USA).

DNA amplification for individuals, studied in present work, was done as described in single PCR cycling reactions. All PCR-products were checked by electrophoresis on $2 \%$ agarose gels.

\section{Sample processing}

About 200 ng of each PCR-product were pooled and purified with the QIAquick PCR purification kit (Qiagen) 
according to the manufacturer's instructions. Biotin 3'end labeling was performed as described [8]. In brief, the eluate resulting from the purification step was dried in a vacuum concentrator and the pellet was dissolved in $5 \mu \mathrm{l}$ of water. Labeling was performed in a total volume of 10 $\mu \mathrm{l}$ with $2.5 \mathrm{U}$ terminal transferase (Roche, Mannheim, Germany), $0.1 \mathrm{mmol} / \mathrm{L}$ Biotin-N6-ddATP (PerkinElmer, Rodgau, Germany), $2.5 \mathrm{nmol} / \mathrm{l} \mathrm{CoCl}_{2}$ and $1 \times$ reaction buffer (Roche). After $1 \mathrm{~h}$ incubation at $37^{\circ} \mathrm{C}$, the enzyme was inactivated at $99^{\circ} \mathrm{C}$ for $15 \mathrm{~min}$ and the mixture cooled on ice.

The final sample hybridization cocktail was made of $10 \mu \mathrm{l}$ of this labeling reaction, $4 \mu \mathrm{l}$ herring sperm DNA $(0.1 \mathrm{mg} /$ $\mathrm{mL}$ with $0.5 \mathrm{mg} / \mathrm{ml}$ BSA), $9 \mu \mathrm{l} 2 \times 2$-[N-morpholino]ethanesulfonic (MES) acid buffer [54.8 mmol/l MES (free acid monohydrate), $147.7 \mathrm{mmol} / \mathrm{l}$ MES sodium salt, $1.8 \mathrm{~mol} / \mathrm{l} \mathrm{NaCl}, 40 \mathrm{mmol} / \mathrm{l} \mathrm{Na}{ }_{2}$ EDTA, $0.02 \%$ (v/v) Tween 20] and $6 \mu \mathrm{l}$ water. As an internal hybridization control, $1 \mu \mathrm{l}$ of a mixture of biotin- $(50 \mathrm{nmol} / \mathrm{l})$ and Cy5labeled $(250 \mathrm{nmol} / \mathrm{l})$ control oligonucleotides (GCAGTGCTGCCATAACCATGAGTGA, CGCAAACTATTAACTGGCGAACTAC， GAACTGGATCTCAACAGCGGTAAGA， AAGATCAGTTGGGTGCACGAGTGGG， CGCAACAATTAATAGACTGGATGG, GCAGTGCTGCCAAAACCATGAGTGA), supplied by febit biotech, were added. The total volume of the hybridization mixture was $30 \mu \mathrm{l}$, which was stored frozen until use at $-20^{\circ} \mathrm{C}$.

\section{Hybridization and detection}

The DNA-fragments of all SNPs of one individual person were analyzed simultaneously in a single hybridization. The biotin-labeled PCR-products in the hybridization mixture were denatured at $99^{\circ} \mathrm{C}$ for $5 \mathrm{~min}$ and quickly cooled on ice for $2 \mathrm{~min}$. The probe arrays were incubated with $1 \times$ MES solution (containing 1\% BSA) at room temperature for $15 \mathrm{~min}$. Then the hybridization mixture was loaded to the array. Hybridization was performed at $45^{\circ} \mathrm{C}$ for $4 \mathrm{~h}$. Subsequently, the used sample was recovered and the array was washed with $0.5 \times$ SSPE buffer (diluted from $6 \times$ SSPE stock-solution consisting of $0.9 \mathrm{~mol} / \mathrm{l} \mathrm{NaCl}, 60$ $\mathrm{mmol} / \mathrm{l} \mathrm{NaH} \mathrm{PO}_{4}\left(\mathrm{pH} 7.4\right.$ ) and $6 \mathrm{mmol} / \mathrm{l} \mathrm{Na} \mathrm{NaDTA}_{2}$ at $45^{\circ} \mathrm{C}$. Staining was performed with $4 \mathrm{ml}$ of $2.5 \mu \mathrm{g} / \mathrm{ml}$ streptavidin R-phycoerythrin conjugate (Molecular Probes, Cologne, Germany) in $6 \times$ SSPE at room temperature for 10 minutes. All these steps were carried out automatically by the Geniom One instrument.

\section{Data analysis}

Image analysis was done automatically with the GeniomOne system-embedded CCD imaging system. All steps such as configuration of detection parameters, acquisition of array image, detection of feature position, calculation of signal intensity and data export to a database were performed automatically. The pattern recogni- tion rules are digitally encoded in the analysis software, simplifying and shortening the result reading. Raw data were further processed with the integrated analysis software with the default settings.

\section{Statistical analysis of epidemiological data}

The analysis was performed with SAS software PHREG version 9 (SAS Institute, Cary, USA). Relative risk of the elevated IgE phenotype associated with genetic variants was estimated by odds ratios (OR) and associated 95 percent confidence limits using the procedure for conditional logistic regression. The gene variants were computed as simultaneous limits of the parameters of a multinomial distribution according to Nieters et al. [23].

\section{Authors' contributions}

JP designed and participated in the experiments and drafted the manuscript. RF contributed to the design of the experiments, conducted genotyping and imaging analysis. $\mathrm{NB}, \mathrm{MB}$ and $\mathrm{AM}$ participated in the design and coordination of the study, contributed to the design of the experiments, conducted SNP selection and statistical analysis and in the preparation of the manuscript. JDH, AM contributed to the design of the experiments and in the preparation of the manuscript. All authors read and approved the final manuscript.

\section{Acknowledgements}

We thank Ina Koegel, Benjamin Heinzerling, Sandra Widder, and Jochen Rudolph for technical support. This study was partially supported by the Estonian Ministry of Education core grant no. 0182582 s03 and the MolTools project funded by the European Commission.

\section{References}

I. A haplotype map of the human genome. Nature 2005, 437:1299-1320.

2. Gray IC, Campbell DA, Spurr NK: Single nucleotide polymorphisms as tools in human genetics. Hum Mol Genet 2000, 9:2403-2408.

3. Ott J, Hoh J: Statistical multilocus methods for disequilibrium analysis in complex traits. Hum Mutat 200I, 17:285-288.

4. Goldstein DB, Cavalleri GL: Genomics: understanding human diversity. Nature 2005, 437: $124 \mid$ - 1242.

5. The Wellcome Trust Case Control Consortium: Genome-wide association study of 14,000 cases of seven common diseases and 3,000 shared controls. Nature 2007, 447:66I-678.

6. Simon R, Radmacher MD, Dobbin K: Design of studies using DNA microarrays. Genet Epidemiol 2002, 23:2I-36.

7. Singh-Gasson S, Green RD, Yue Y, Nelson C, Blattner F, Sussman MR, Cerrina F: Maskless fabrication of light-directed oligonucleotide microarrays using a digital micromirror array. Nat Biotechnol 1999, 17:974-978.

8. Baum M, Bielau S, Rittner N, Schmid K, Eggelbusch K, Dahms M, Schlauersbach A, Tahedl H, Beier M, Guimil R, Scheffler M, Hermann C, Funk JM, Wixmerten A, Rebscher H, Honig M, Andreae C, Buchner D, Moschel E, Glathe A, Jager E, Thom M, Greil A, Bestvater F, Obermeier F, Burgmaier J, Thome K, Weichert S, Hein S, Binnewies T, Foitzik V, Muller M, Stahler CF, Stahler PF: Validation of a novel, fully integrated and flexible microarray benchtop facility for gene expression profiling. Nucleic Acids Res 2003, 3 I :e I 5 I.

9. Beier M, Hoheisel JD: Production by quantitative photolithographic synthesis of individually quality checked DNA microarrays. Nucleic Acids Res 2000, 28:EII. 
10. Nieters A, Linseisen J, Becker N: Association of polymorphisms in Th I, Th2 cytokine genes with hayfever and atopy in a subsample of EPIC-Heidelberg. Clin Exp Allergy 2004, 34:346-353.

II. dbSNP [http://www.ncbi.nlm.nih.gov/SNP/]

12. HGVBASE [http://hgvbase.cgb.ki.se/]

13. Genecanvas [http://genecanvas.idf.inserm.fr/]

14. Dai H, Meyer M, Stepaniants S, Ziman M, Stoughton R: Use of hybridization kinetics for differentiating specific from nonspecific binding to oligonucleotide microarrays. Nucleic Acids Res 2002, 30:e86.

15. Wetmur JG, Davidson N: Kinetics of renaturation of DNA. J Mol Biol 1968, 31:349-370

16. Breslauer KJ, Frank R, Blocker H, Marky LA: Predicting DNA duplex stability from the base sequence. Proc Natl Acad Sci U S A 1986, 83:3746-3750.

17. Southern E, Mir K, Shchepinov M: Molecular interactions on microarrays. Nat Genet 1999, 2 1:5-9.

18. Jobs M, Howell WM, Stromqvist L, Mayr T, Brookes AJ: DASH-2: flexible, low-cost, and high-throughput SNP genotyping by dynamic allele-specific hybridization on membrane arrays. Genome Res 2003, 13:916-924.

19. Maskos U, Southern EM: Parallel analysis of oligodeoxyribonucleotide (oligonucleotide) interactions. I. Analysis of factors influencing oligonucleotide duplex formation. Nucleic Acids Res 1992, 20:1675-1678.

20. Williams JC, Case-Green SC, Mir KU, Southern EM: Studies of oligonucleotide interactions by hybridisation to arrays: the influence of dangling ends on duplex yield. Nucleic Acids Res 1994, 22: 1365-1367.

21. Daser A, Koetz K, Batjer N, Jung M, Ruschendorf F, Goltz M, Ellerbrok $\mathrm{H}$, Renz $\mathrm{H}$, Walter J, Paulsen M: Genetics of atopy in a mouse model: polymorphism of the IL-5 receptor alpha chain. Immunogenetics 2000, 51:632-638.

22. Primer3 [http://www.genome.wi.mit.edu/cgi-bin/primer/ primer3 www.cgi]

23. Nieters A, Brems S, Becker N: Cross-sectional study on cytokine polymorphisms, cytokine production after $\mathrm{T}$-cell stimulation and clinical parameters in a random sample of a German population. Hum Genet 200I, 108:24I-248.
Publish with Bio Med Central and every scientist can read your work free of charge

"BioMed Central will be the most significant development for disseminating the results of biomedical research in our lifetime. "

Sir Paul Nurse, Cancer Research UK

Your research papers will be:

- available free of charge to the entire biomedical community

- peer reviewed and published immediately upon acceptance

- cited in PubMed and archived on PubMed Central

- yours - you keep the copyright

Submit your manuscript here:

http://www.biomedcentral.com/info/publishing_adv.asp
BioMedcentral 